\title{
THE BEST CONSTANT OF SOBOLEV INEQUALITY CORRESPONDING TO A BENDING PROBLEM OF A BEAM ON AN INTERVAL
}

\author{
By \\ Kazuo Takemura, Hiroyuki Yamagishi, Yoshinori Kametaka, \\ Kohtaro Watanabe and Atsushi NagaI
}

\begin{abstract}
Green function of 2-point simple-type self-adjoint boundary value problem for 4-th order linear ordinary differential equation, which represents bending of a beam with the boundary condition as clamped, Dirichlet, Neumann and free. The construction of Green function needs the symmetric orthogonalization method in some cases. Green function is the reproducing kernel for suitable set of Hilbert space and inner product. As an application, the best constants of the corresponding Sobolev inequalities are expressed as the maximum of the diagonal values of Green function.
\end{abstract}

\section{Preparation}

A beam is supported by uniformly distributed springs with spring constant $q>0$ on a fixed floor and is exerted a tension $p>0$ on both sides. Under a density of a load $f(x)$, a bending of a beam $u(x)$ [11] satisfies the following 4-th order linear ordinary differential equation [2]: $u^{(4)}-p u^{\prime \prime}+q u=f(x)$ $(-1<x<1)$. In this paper, we consider the boundary value problem for bending of a beam on an interval in the degenerate case $p=q=0$ :

$$
\begin{aligned}
& \operatorname{BVP}(\alpha, \beta) \\
& \left\{\begin{array}{l}
u^{(4)}=f(x) \quad(-1<x<1) \\
u^{\left(\alpha_{i}\right)}(-1)=u^{\left(\beta_{i}\right)}(1)=0 \quad(i=0,1)
\end{array}\right.
\end{aligned}
$$

2000 Mathematics Subject Classification: Primary 34B27, Secondary 46E35, 41A44.

Key words and phrases: Green function, Sobolev inequality, best constant, reproducing kernel, symmetric orthogonalization method.

Received April 1, 2009.

Revised August 3, 2009. 
where $\alpha=\left(\alpha_{0}, \alpha_{1}\right)$ and $\beta=\left(\beta_{0}, \beta_{1}\right)$ take 6 different values $(0,1),(0,2),(0,3)$, $(1,2),(1,3),(2,3)$. Among them, we here treat only self-adjoint cases $\alpha, \beta=$ $(0,1),(0,2),(1,3),(2,3)$, which also have engineering importance and correspond to clamped, Dirichlet (simply-supported), Neumann (sliding) and free edge, respectively [8, Chap. 2]. Therefore, the following 16 kinds of $(\alpha, \beta)$

$$
\begin{aligned}
(\alpha, \beta)= & (0,1,0,1),(0,1,0,2),(0,1,1,3),(0,1,2,3), \\
& (0,2,0,1),(0,2,0,2),(0,2,1,3),(0,2,2,3), \\
& (1,3,0,1),(1,3,0,2),(1,3,1,3),(1,3,2,3), \\
& (2,3,0,1),(2,3,0,2),(2,3,1,3),(2,3,2,3)
\end{aligned}
$$

can be considered. However, throughout this paper, we focus our attention only on $10(\alpha, \beta)$ among them,

$$
\begin{aligned}
& (\alpha, \beta)=(0,1,0,1),(0,1,0,2),(0,1,1,3),(0,1,2,3), \\
& (0,2,0,2),(0,2,1,3),(0,2,2,3) \text {, } \\
& (1,3,1,3),(1,3,2,3) \text {, } \\
& (2,3,2,3)
\end{aligned}
$$

taking account of the symmetry. The eigen value problem:

$$
\begin{aligned}
& \operatorname{EVP}(\alpha, \beta) \\
& \left\{\begin{array}{l}
u^{(4)}=\lambda u \quad(-1<x<1) \\
u^{\left(\alpha_{i}\right)}(-1)=u^{\left(\beta_{i}\right)}(1)=0 \quad(i=0,1)
\end{array}\right.
\end{aligned}
$$

has eigen function corresponding to $\lambda=0$ in some cases. In these cases, the additional conditions are required for the uniqueness and existence of the solution to $\operatorname{BVP}(\alpha, \beta)$. If $(\alpha, \beta)=(0,1,0,1),(0,1,0,2),(0,1,1,3),(0,1,2,3),(0,2,0,2)$, $(0,2,1,3)$, then $\lambda=0$ is not an eigenvalue. If $(\alpha, \beta)=(0,2,2,3),(1,3,1,3)$, $(1,3,2,3)$, then $\lambda=0$ is an eigenvalue and the corresponding eigenspace is onedimensional. If $(\alpha, \beta)=(2,3,2,3)$, then $\lambda=0$ is an eigenvalue and the corresponding eigenspace is two-dimensional. The normalized eigenfunction $\varphi(x)=$ $\varphi(\alpha, \beta ; x)(-1<x<1)$ is given by

$$
\varphi(0,2,2,3 ; x)=\sqrt{\frac{3}{8}}(1+x), \quad \varphi(1,3,1,3 ; x)=\varphi(1,3,2,3 ; x)=\frac{1}{\sqrt{2}}
$$

and $\varphi_{i}(x)=\varphi_{i}(2,3,2,3 ; x)(i=0,1,-1<x<1)$ are given by

$$
\varphi_{0}(x)=\frac{1}{\sqrt{2}}, \quad \varphi_{1}(x)=\sqrt{\frac{3}{2}} x .
$$


We prepare the solvability condition

$$
(\mathbf{S}):\left\{\begin{array}{lrl}
\text { none } & (\alpha, \beta)= & (0,1,0,1),(0,1,0,2),(0,1,1,3), \\
& (0,1,2,3),(0,2,0,2),(0,2,1,3) \\
\int_{-1}^{1} f(y) \varphi(y) d y=0 & (\alpha, \beta)= & (0,2,2,3),(1,3,1,3),(1,3,2,3) \\
\int_{-1}^{1} f(y) \varphi_{i}(y) d y=0 & (i=0,1) & (\alpha, \beta)=(2,3,2,3)
\end{array}\right.
$$

and the orthogonality condition

$$
(\mathrm{O}):\left\{\begin{array}{lr}
\text { none } & (\alpha, \beta)=(0,1,0,1),(0,1,0,2),(0,1,1,3), \\
& (0,1,2,3),(0,2,0,2),(0,2,1,3) \\
\int_{-1}^{1} u(x) \varphi(x) d x=0 & (\alpha, \beta)=(0,2,2,3),(1,3,1,3),(1,3,2,3) \\
\int_{-1}^{1} u(x) \varphi_{i}(x) d x=0 & (i=0,1)
\end{array}\right.
$$

Concerning the uniqueness and existence of the solution to $\operatorname{BVP}(\alpha, \beta)$, we have obtained the following theorem:

THEOREM 1.1. (1) For any bounded continuous function $f(x)$ on an interval $-1<x<1$ satisfying $(\mathrm{S}), \operatorname{BVP}(\alpha, \beta)$ with $(\mathrm{O})$ has a unique classical solution $u(x)$ expressed as

$$
u(x)=\int_{-1}^{1} G(x, y) f(y) d y \quad(-1<x<1)
$$

where $G(x, y)=G(\alpha, \beta ; x, y)$ is Green function.

(2) Green functions $G(x, y)=G(\alpha, \beta ; x, y)(-1<x, y<1)$ are given as follows:

$$
\begin{aligned}
G(0,1,0,1 ; x, y)= & \frac{1}{12}|x-y|^{3}+\frac{1}{24}\left[-x^{3} y^{3}+3\left(x^{3} y+x y^{3}\right)\right. \\
& \left.-3 x^{2} y^{2}-3\left(x^{2}+y^{2}\right)+3 x y+1\right]
\end{aligned}
$$




$$
\begin{aligned}
G(0,1,0,2 ; x, y)= & \frac{1}{12}|x-y|^{3}+\frac{1}{96}\left[-x^{3} y^{3}+3\left(x^{3} y^{2}+x^{2} y^{3}\right)+9\left(x^{3} y+x y^{3}\right)\right. \\
& -9 x^{2} y^{2}-3\left(x^{3}+y^{3}\right)-3\left(x^{2} y+x y^{2}\right)-15\left(x^{2}+y^{2}\right) \\
& +15 x y+3(x+y)+7]
\end{aligned}
$$

$G(0,1,1,3 ; x, y)=\frac{1}{12}|x-y|^{3}+\frac{1}{24}\left[-3 x^{2} y^{2}-2\left(x^{3}+y^{3}\right)-3\left(x^{2}+y^{2}\right)\right.$

$$
+12 x y+6(x+y)+5]
$$

$$
\begin{aligned}
G(0,1,2,3 ; x, y)= & \frac{1}{12}\left[|x-y|^{3}-\left(x^{3}+y^{3}\right)+3\left(x^{2} y+x y^{2}\right)\right. \\
& +12 x y+6(x+y)+4]
\end{aligned}
$$

$$
G(0,2,0,2 ; x, y)=\frac{1}{12}\left[|x-y|^{3}+x^{3} y+x y^{3}-3\left(x^{2}+y^{2}\right)+2 x y+2\right]
$$

$$
\begin{aligned}
G(0,2,1,3 ; x, y)= & \frac{1}{12}\left[|x-y|^{3}-\left(x^{3}+y^{3}\right)-3\left(x^{2} y+x y^{2}\right)-6\left(x^{2}+y^{2}\right)\right. \\
& +12 x y+12(x+y)+16]
\end{aligned}
$$

$$
\begin{aligned}
G(0,2,2,3 ; x, y)= & \frac{1}{12}|x-y|^{3}+\frac{1}{6720}\left[-21\left(x^{5} y+x y^{5}\right)-21\left(x^{5}+y^{5}\right)\right. \\
& -105\left(x^{4} y+x y^{4}\right)-105\left(x^{4}+y^{4}\right)+630\left(x^{3} y+x y^{3}\right) \\
& +70\left(x^{3}+y^{3}\right)+630\left(x^{2} y+x y^{2}\right)-1050\left(x^{2}+y^{2}\right) \\
& +1278 x y-318(x+y)+326]
\end{aligned}
$$

$G(1,3,1,3 ; x, y)=\frac{1}{12}|x-y|^{3}-\frac{1}{48}\left[x^{4}+y^{4}+6 x^{2} y^{2}\right.$

$$
\left.+4\left(x^{2}+y^{2}\right)-24 x y\right]+\frac{1}{90}
$$

$$
\begin{aligned}
G(1,3,2,3 ; x, y)= & \frac{1}{12}|x-y|^{3}+\frac{1}{48}\left[-\left(x^{4}+y^{4}\right)+12\left(x^{2} y+x y^{2}\right)\right. \\
& \left.-6\left(x^{2}+y^{2}\right)+48 x y-4(x+y)\right]+\frac{1}{40}
\end{aligned}
$$

$$
\begin{aligned}
G(2,3,2,3 ; x, y)= & \frac{1}{12}|x-y|^{3}-\frac{1}{1680}\left[21\left(x^{5} y+x y^{5}\right)+35\left(x^{4}+y^{4}\right)\right. \\
& \left.-210\left(x^{3} y+x y^{3}\right)+210\left(x^{2}+y^{2}\right)-198 x y\right]+\frac{1}{40}
\end{aligned}
$$


This theorem is shown in section 4. The above bending problem of a beam is important in the field of classical mechanics of materials. The purpose of this paper is to give a mathematical foundation of this problem.

\section{Conclusion}

Let us introduce Sobolev space

$$
\begin{aligned}
& H=H(\alpha, \beta)=\left\{u(x) \mid u(x), u^{\prime \prime}(x) \in L^{2}(-1,1), A(\alpha, \beta)\right\} \\
& A(0,1,0,1): u(-1)=u^{\prime}(-1)=u(1)=u^{\prime}(1)=0 \\
& A(0,1,0,2): u(-1)=u^{\prime}(-1)=u(1)=0 \\
& A(0,1,1,3): u(-1)=u^{\prime}(-1)=u^{\prime}(1)=0 \\
& A(0,1,2,3): u(-1)=u^{\prime}(-1)=0 \\
& A(0,2,0,2): u(-1)=u(1)=0 \\
& A(0,2,1,3): u(-1)=u^{\prime}(1)=0 \\
& A(0,2,2,3): u(-1)=0, \quad \int_{-1}^{1} u(x) \varphi(x) d x=0 \\
& A(1,3,1,3): u^{\prime}(-1)=u^{\prime}(1)=0, \quad \int_{-1}^{1} u(x) \varphi(x) d x=0 \\
& A(1,3,2,3): u^{\prime}(-1)=0, \quad \int_{-1}^{1} u(x) \varphi(x) d x=0 \\
& A(2,3,2,3): \int_{-1}^{1} u(x) \varphi_{i}(x) d x=0 \quad(i=0,1),
\end{aligned}
$$

and Sobolev inner product

$$
(u, v)_{H}=\int_{-1}^{1} u^{\prime \prime}(x) \bar{v}^{\prime \prime}(x) d x, \quad\|u\|_{H}^{2}=(u, u)_{H}=\int_{-1}^{1}\left|u^{\prime \prime}(x)\right|^{2} d x .
$$

Here $^{\prime}$ is a derivative in a distributional sense. So, any element $u \in H$ belongs to $C^{1}[-1,1]$ from Sobolev embedding theorem; see [1, Chap. VIII. 2]. $(\cdot, \cdot)_{H}$ is proved to be an inner product of $H$ afterwards. $H$ is Hilbert space with an inner product $(\cdot, \cdot)_{H}$. We here present main conclusion in this paper. 
THEOREM 2.1. (1) For any function $u(x) \in H(\alpha, \beta)$, there exists a positive constant $C$ which is independent of $u(x)$ such that Sobolev inequality

$$
\left(\sup _{|y| \leq 1}|u(y)|\right)^{2} \leq C \int_{-1}^{1}\left|u^{\prime \prime}(x)\right|^{2} d x
$$

holds. Among such $C$ the best constant $C(\alpha, \beta)$ is

$$
C(\alpha, \beta)=\max _{|y| \leq 1} G(\alpha, \beta ; y, y)=G\left(\alpha, \beta ; y_{0}, y_{0}\right)
$$

where $y_{0}$ satisfies $\left|y_{0}\right| \leq 1$. If we replace $C$ by $C(\alpha, \beta)$ in (2.3), equality holds for $u(x)=c G\left(\alpha, \beta ; x, y_{0}\right)(-1<x<1)$ for every complex number $c$.

(2) Concrete forms of $C(\alpha, \beta)$ are given as follows:

$$
\begin{aligned}
C(0,1,0,1) & =\max _{|y| \leq 1} G(0,1,0,1 ; y, y)=G(0,1,0,1 ; 0,0)=\frac{1}{24} \\
C(0,1,0,2) & =\max _{|y| \leq 1} G(0,1,0,2 ; y, y)=G(0,1,0,2 ; 3-2 \sqrt{2}, 3-2 \sqrt{2}) \\
& =\frac{8}{3}(17-12 \sqrt{2}) \\
C(0,1,1,3) & =\max _{|y| \leq 1} G(0,1,1,3 ; y, y)=G(0,1,1,3 ; 1,1)=\frac{2}{3} \\
C(0,1,2,3) & =\max _{|y| \leq 1} G(0,1,2,3 ; y, y)=G(0,1,2,3 ; 1,1)=\frac{8}{3} \\
C(0,2,0,2) & =\max _{|y| \leq 1} G(0,2,0,2 ; y, y)=G(0,2,0,2 ; 0,0)=\frac{1}{6} \\
C(0,2,1,3) & =\max _{|y| \leq 1} G(0,2,1,3 ; y, y)=G(0,2,1,3 ; 1,1)=\frac{8}{3} \\
C(0,2,2,3) & =\max _{|y| \leq 1} G(0,2,2,3 ; y, y)=G(0,2,2,3 ; 1,1)=\frac{16}{105} \\
C(1,3,1,3) & =\max _{|y| \leq 1} G(1,3,1,3 ; y, y)=G(1,3,1,3 ;-1,-1) \\
C(1,3,2,3) & =\max _{|y| \leq 1} G(1,3,2,3 ; y, y)=G(1,3,2,3 ; 1,1)=\frac{16}{15} \\
& =6
\end{aligned}
$$




$$
\begin{aligned}
C(2,3,2,3) & =\max _{|y| \leq 1} G(2,3,2,3 ; y, y)=G(2,3,2,3 ;-1,-1) \\
& =G(2,3,2,3 ; 1,1)=\frac{8}{105} .
\end{aligned}
$$

The engineering meaning of Sobolev inequality is that the square of the maximum bending of a beam $u(y)$ is estimated from above by the constant multiple of the potential energy $\|u\|_{H}$. Among these constants, the best constant is the maximum of the diagonal value of the impulse response $G(x, y)$. If boundary condition becomes looser as $(0,1) \rightarrow(0,2) \rightarrow(1,3) \rightarrow(2,3)$, the impulse response $G(x, y)$ gets larger especially on the boundary. Therefore, the diagonal value of Green function attains its maximum at the boundary $(y=-1$ or 1$)$ in the case of $(\alpha, \beta)=(0,1,1,3),(0,1,2,3),(0,2,1,3),(0,2,2,3),(1,3,1,3),(1,3,2,3),(2,3,2,3)$. On the other hand, if $(\alpha, \beta)=(0,1,0,1),(0,1,0,2),(0,2,0,2)$, the case of strong restriction, the maximums are attained at or near the center point $(y=0)$.

We have already obtained the best constant of Sobolev inequality for $(d / d x)^{4}$ in the case of $(\alpha, \beta)=(0,2,0,2)[5,6,13],(\alpha, \beta)=(0,2,1,3)[13], \quad(\alpha, \beta)=$ $(1,3,1,3)[5,13]$ and $(\alpha, \beta)=(2,3,2,3)[10]$.

This paper is composed of seven sections. In section 3, we state boundary value problem for bending of a beam. In section 4, Theorem 1.1 is proved. In particular, we construct Green function by the method of symmetric orthogonalization in some special cases. In section 5, we show Green function is the reproducing kernel for $H$ and $(\cdot, \cdot)_{H}$. Finally in section 6 and 7 , we prove Theorem 2.1(1) and (2), respectively.

\section{Boundary Value Problems}

We introduce functions $K_{j}(x)$ defined by

$$
K_{j}(x)=\frac{x^{-j+3}}{(-j+3) !} \quad(j=\cdots,-1,0,1,2,3), \quad 0 \quad(j \geq 4),
$$

which satisfy recurrence relation $K_{j}^{\prime}(x)=K_{j+1}(x)$. We adopt the abbreviation $K_{j}=K_{j}(2)$ and note that $K_{j}(0)=0(j \neq 3), 1 \quad(j=3)$.

In order to explain the meaning of the solvability condition (1.7) and the orthogonality condition (1.8), we show the following theorem.

THEOREM 3.1.

CASE I $(\alpha, \beta)=(0,1,0,1),(0,1,0,2),(0,1,1,3), \quad(0,1,2,3), \quad(0,2,0,2)$, $(0,2,1,3)$ 
For any bounded continuous function $f(x)$ on an interval $-1<x<1$, $\operatorname{BVP}(\alpha, \beta)$ has a classical solution $u(x)$ expressed as

$$
u(x)=\int_{-1}^{1} G(\alpha, \beta ; x, y) f(y) d y \quad(-1<x<1) .
$$

Green functions $G(\alpha, \beta ; x, y)$ are given by

$$
\begin{aligned}
& G(\alpha, \beta ; x, y) \\
& =\frac{1}{2}\left[K_{0}(|x-y|)+\kappa^{-1}\left\{\left|\begin{array}{cc|c}
K_{\alpha_{0}+\beta_{0}} & K_{\alpha_{1}+\beta_{0}} & K_{\beta_{0}}(1-y) \\
K_{\alpha_{0}+\beta_{1}} & K_{\alpha_{1}+\beta_{1}} & K_{\beta_{1}}(1-y) \\
\hline K_{\alpha_{0}}(1+x) & K_{\alpha_{1}}(1+x) & 0
\end{array}\right|\right.\right. \\
& \left.+\left|\begin{array}{cc|c}
K_{\alpha_{0}+\beta_{0}} & K_{\alpha_{1}+\beta_{0}} & K_{\beta_{0}}(1-x) \\
K_{\alpha_{0}+\beta_{1}} & K_{\alpha_{1}+\beta_{1}} & K_{\beta_{1}}(1-x) \\
\hline K_{\alpha_{0}}(1+y) & K_{\alpha_{1}}(1+y) & 0
\end{array}\right|\right\} \quad(-1<x, y<1)
\end{aligned}
$$

where $\kappa=\left|\begin{array}{ll}K_{\alpha_{0}+\beta_{0}} & K_{\alpha_{1}+\beta_{0}} \\ K_{\alpha_{0}+\beta_{1}} & K_{\alpha_{1}+\beta_{1}}\end{array}\right|<0$.

CASE II $(\alpha, \beta)=(0,2,2,3),(1,3,1,3),(1,3,2,3)$

Under the solvability condition

$$
\int_{-1}^{1} f(y) \varphi(\alpha, \beta ; y) d y=0
$$

$u(x)$ is given as

$$
u(x)=\int_{-1}^{1} G_{0}(\alpha, \beta ; x, y) f(y) d y+c \varphi(\alpha, \beta ; x) \quad(-1<x<1)
$$

where $c$ is an arbitrary constant and $G_{0}(\alpha, \beta ; x, y)$ are given by

$$
\begin{aligned}
G_{0}(\alpha, \beta ; x, y)= & \frac{1}{2}\left[K_{0}(|x-y|)+K_{\alpha_{0}+\beta_{0}}^{-1}\left\{\left|\begin{array}{c|c}
K_{\alpha_{0}+\beta_{0}} & K_{\beta_{0}}(1-y) \\
K_{\alpha_{0}}(1+x) & 0
\end{array}\right|\right.\right. \\
+ & \left.\left.\mid \begin{array}{c|c}
K_{\alpha_{0}+\beta_{0}} & K_{\beta_{0}}(1-x) \\
\hline K_{\alpha_{0}}(1+y) & 0
\end{array}\right\}\right] \quad(-1<x, y<1) \\
G_{0}(0,2,2,3 ; x, y)= & \frac{1}{12}|x-y|^{3}+\frac{1}{24}\left[x^{3} y+x y^{3}-\left(x^{3}+y^{3}\right)+3\left(x^{2} y+x y^{2}\right)\right. \\
& \left.-3\left(x^{2}+y^{2}\right)+6 x y-2(x+y)-2\right]
\end{aligned}
$$




$$
\begin{aligned}
G_{0}(1,3,1,3 ; x, y)= & \frac{1}{12}|x-y|^{3}-\frac{1}{8}\left[x^{2} y^{2}+x^{2}+y^{2}-4 x y+1\right] \\
G_{0}(1,3,2,3 ; x, y)= & \frac{1}{12}|x-y|^{3}+\frac{1}{4}\left[x^{2} y+x y^{2}-\left(x^{2}+y^{2}\right)\right. \\
& +4 x y-(x+y)-2]
\end{aligned}
$$

which are called the proto Green functions.

CASE III $(\alpha, \beta)=(2,3,2,3)$

Under the solvability condition

$$
\int_{-1}^{1} f(y) \varphi_{i}(y) d y=0 \quad(i=0,1)
$$

$u(x)$ is given as

$$
u(x)=\int_{-1}^{1} G_{0}(2,3,2,3 ; x, y) f(y) d y+c_{0} \varphi_{0}(x)+c_{1} \varphi_{1}(x) \quad(-1<x<1)
$$

where $c_{0}$ and $c_{1}$ are arbitrary constants. The proto Green function $G_{0}(2,3,2,3 ; x, y)$ is given by

$$
G_{0}(2,3,2,3 ; x, y)=\frac{1}{2} K_{0}(|x-y|)=\frac{1}{12}|x-y|^{3} \quad(-1<x, y<1) .
$$

Proof of Theorem 3.1. Let us define

$$
\boldsymbol{u}=\left(\begin{array}{l}
u_{0} \\
u_{1} \\
u_{2} \\
u_{3}
\end{array}\right)=\left(\begin{array}{c}
u \\
u^{\prime} \\
u^{\prime \prime} \\
u^{\prime \prime \prime}
\end{array}\right), \quad \boldsymbol{e}=\left(\begin{array}{l}
0 \\
0 \\
0 \\
1
\end{array}\right), \quad \boldsymbol{N}=\left(\begin{array}{llll}
0 & 1 & 0 & 0 \\
0 & 0 & 1 & 0 \\
0 & 0 & 0 & 1 \\
0 & 0 & 0 & 0
\end{array}\right)
$$

then $\operatorname{BVP}(\alpha, \beta)$ is rewritten as

$$
\begin{cases}\boldsymbol{u}^{\prime}=\boldsymbol{N} \boldsymbol{u}+\boldsymbol{e} f(x) & (-1<x<1) \\ u_{\alpha_{i}}(-1)=u_{\beta_{i}}(1)=0 & (i=0,1) .\end{cases}
$$

Let $\boldsymbol{E}(x)$ be expressed as $\boldsymbol{E}(x)=\exp (\boldsymbol{N} x)=\boldsymbol{K}(x) \boldsymbol{K}(0)^{-1}$ where

$$
\boldsymbol{K}(x)=\left(\begin{array}{llll}
K_{0} & K_{1} & K_{2} & K_{3} \\
K_{1} & K_{2} & K_{3} & K_{4} \\
K_{2} & K_{3} & K_{4} & K_{5} \\
K_{3} & K_{4} & K_{5} & K_{6}
\end{array}\right)(x), \quad \boldsymbol{K}(0)^{-1}=\left(\begin{array}{cccc}
0 & 0 & 0 & 1 \\
0 & 0 & 1 & 0 \\
0 & 1 & 0 & 0 \\
1 & 0 & 0 & 0
\end{array}\right)=\boldsymbol{K}(0)
$$


which is a fundamental solution to the initial value problem $\boldsymbol{E}^{\prime}=\boldsymbol{N} \boldsymbol{E}, \boldsymbol{E}(0)=\boldsymbol{I}$. $\boldsymbol{I}$ is a unit matrix. Solving (3.13), we have

$$
\begin{aligned}
& \boldsymbol{u}(x)=\boldsymbol{E}(x+1) \boldsymbol{u}(-1)+\int_{-1}^{x} \boldsymbol{E}(x-y) \boldsymbol{e} f(y) d y \\
& \boldsymbol{u}(x)=\boldsymbol{E}(x-1) \boldsymbol{u}(1)-\int_{x}^{1} \boldsymbol{E}(x-y) \boldsymbol{e} f(y) d y,
\end{aligned}
$$

and equivalently,

$$
\begin{aligned}
& \left(\begin{array}{l}
u_{0} \\
u_{1} \\
u_{2} \\
u_{3}
\end{array}\right)(x)=\left(\begin{array}{llll}
K_{0} & K_{1} & K_{2} & K_{3} \\
K_{1} & K_{2} & K_{3} & K_{4} \\
K_{2} & K_{3} & K_{4} & K_{5} \\
K_{3} & K_{4} & K_{5} & K_{6}
\end{array}\right)(x+1)\left(\begin{array}{l}
u_{3} \\
u_{2} \\
u_{1} \\
u_{0}
\end{array}\right)(-1)+\int_{-1}^{x}\left(\begin{array}{l}
K_{0} \\
K_{1} \\
K_{2} \\
K_{3}
\end{array}\right)(x-y) f(y) d y \\
& \left(\begin{array}{l}
u_{0} \\
u_{1} \\
u_{2} \\
u_{3}
\end{array}\right)(x)=\left(\begin{array}{llll}
K_{0} & K_{1} & K_{2} & K_{3} \\
K_{1} & K_{2} & K_{3} & K_{4} \\
K_{2} & K_{3} & K_{4} & K_{5} \\
K_{3} & K_{4} & K_{5} & K_{6}
\end{array}\right)(x-1)\left(\begin{array}{l}
u_{3} \\
u_{2} \\
u_{1} \\
u_{0}
\end{array}\right)(1)-\int_{x}^{1}\left(\begin{array}{l}
K_{0} \\
K_{1} \\
K_{2} \\
K_{3}
\end{array}\right)(x-y) f(y) d y .
\end{aligned}
$$

Comparing 0-th row, we have

$$
\begin{aligned}
u_{0}(x)= & \left(K_{\alpha_{0}}, K_{\alpha_{1}}\right)(x+1)\left(\begin{array}{l}
u_{3-\alpha_{0}} \\
u_{3-\alpha_{1}}
\end{array}\right)(-1)+\left(K_{3-\alpha_{0}}, K_{3-\alpha_{1}}\right)(x+1)\left(\begin{array}{l}
u_{\alpha_{0}} \\
u_{\alpha_{1}}
\end{array}\right)(-1) \\
& +\int_{-1}^{x} K_{0}(x-y) f(y) d y \\
u_{0}(x)= & \left(K_{\beta_{0}}, K_{\beta_{1}}\right)(x-1)\left(\begin{array}{c}
u_{3-\beta_{0}} \\
u_{3-\beta_{1}}
\end{array}\right)(1)+\left(K_{3-\beta_{0}}, K_{3-\beta_{1}}\right)(x-1)\left(\begin{array}{l}
u_{\beta_{0}} \\
u_{\beta_{1}}
\end{array}\right)(1) \\
& -\int_{x}^{1} K_{0}(x-y) f(y) d y .
\end{aligned}
$$

Employing the boundary conditions (3.14), we have

$$
\begin{aligned}
& u_{0}(x)=\left(K_{\alpha_{0}}, K_{\alpha_{1}}\right)(x+1)\left(\begin{array}{l}
u_{3-\alpha_{0}} \\
u_{3-\alpha_{1}}
\end{array}\right)(-1)+\int_{-1}^{x} K_{0}(x-y) f(y) d y \\
& u_{0}(x)=\left(K_{\beta_{0}}, K_{\beta_{1}}\right)(x-1)\left(\begin{array}{l}
u_{3-\beta_{0}} \\
u_{3-\beta_{1}}
\end{array}\right)(1)-\int_{x}^{1} K_{0}(x-y) f(y) d y .
\end{aligned}
$$

Noting $K_{0}(x-y)=-K_{0}(y-x)$ and taking an average of (3.15) and (3.16), we have 


$$
\begin{aligned}
u_{0}(x)= & \frac{1}{2}\left(K_{\alpha_{0}}, K_{\alpha_{1}}\right)(x+1)\left(\begin{array}{c}
u_{3-\alpha_{0}} \\
u_{3-\alpha_{1}}
\end{array}\right)(-1)+\frac{1}{2}\left(K_{\beta_{0}}, K_{\beta_{1}}\right)(x-1)\left(\begin{array}{c}
u_{3-\beta_{0}} \\
u_{3-\beta_{1}}
\end{array}\right)(1) \\
& +\int_{-1}^{1} \frac{1}{2} K_{0}(|x-y|) f(y) d y .
\end{aligned}
$$

Taking $\beta_{i}$-th derivative for (3.15) and $\alpha_{i}$-th derivative for (3.16), we obtain

$$
\begin{aligned}
& u_{\beta_{i}}(x)=\left(K_{\alpha_{0}+\beta_{i}}, K_{\alpha_{1}+\beta_{i}}\right)(x+1)\left(\begin{array}{l}
u_{3-\alpha_{0}} \\
u_{3-\alpha_{1}}
\end{array}\right)(-1)+\int_{-1}^{x} K_{\beta_{i}}(x-y) f(y) d y \\
& u_{\alpha_{i}}(x)=\left(K_{\alpha_{i}+\beta_{0}}, K_{\alpha_{i}+\beta_{1}}\right)(x-1)\left(\begin{array}{c}
u_{3-\beta_{0}} \\
u_{3-\beta_{1}}
\end{array}\right)(1)-\int_{x}^{1} K_{\alpha_{i}}(x-y) f(y) d y .
\end{aligned}
$$

Putting $x=1$ in (3.18) and $x=-1$ in (3.19) and considering the boundary condition (3.14) again, we obtain

$$
\begin{aligned}
& \left(\begin{array}{ll}
K_{\alpha_{0}+\beta_{0}} & K_{\alpha_{1}+\beta_{0}} \\
K_{\alpha_{0}+\beta_{1}} & K_{\alpha_{1}+\beta_{1}}
\end{array}\right)(2)\left(\begin{array}{c}
u_{3-\alpha_{0}} \\
u_{3-\alpha_{1}}
\end{array}\right)(-1)=-\int_{-1}^{1}\left(\begin{array}{c}
K_{\beta_{0}} \\
K_{\beta_{1}}
\end{array}\right)(1-y) f(y) d y \\
& \left(\begin{array}{ll}
K_{\alpha_{0}+\beta_{0}} & K_{\alpha_{0}+\beta_{1}} \\
K_{\alpha_{1}+\beta_{0}} & K_{\alpha_{1}+\beta_{1}}
\end{array}\right)(-2)\left(\begin{array}{l}
u_{3-\beta_{0}} \\
u_{3-\beta_{1}}
\end{array}\right)(1)=\int_{-1}^{1}\left(\begin{array}{c}
K_{\alpha_{0}} \\
K_{\alpha_{1}}
\end{array}\right)(-1-y) f(y) d y .
\end{aligned}
$$

CASE I Since two matrices on the left hand side of (3.20) and (3.21) are confirmed to be invertible, we have

$$
\begin{gathered}
\left(\begin{array}{c}
u_{3-\alpha_{0}} \\
u_{3-\alpha_{1}}
\end{array}\right)(-1)=-\int_{-1}^{1}\left(\begin{array}{ll}
K_{\alpha_{0}+\beta_{0}} & K_{\alpha_{1}+\beta_{0}} \\
K_{\alpha_{0}+\beta_{1}} & K_{\alpha_{1}+\beta_{1}}
\end{array}\right)^{-1}(2)\left(\begin{array}{c}
K_{\beta_{0}} \\
K_{\beta_{1}}
\end{array}\right)(1-y) f(y) d y \\
\left(\begin{array}{c}
u_{3-\beta_{0}} \\
u_{3-\beta_{1}}
\end{array}\right)(1)=\int_{-1}^{1}\left(\begin{array}{ll}
K_{\alpha_{0}+\beta_{0}} & K_{\alpha_{0}+\beta_{1}} \\
K_{\alpha_{1}+\beta_{0}} & K_{\alpha_{1}+\beta_{1}}
\end{array}\right)^{-1}(-2)\left(\begin{array}{c}
K_{\alpha_{0}} \\
K_{\alpha_{1}}
\end{array}\right)(-1-y) f(y) d y .
\end{gathered}
$$

Noting $u=u_{0}$ and substituting above two equalities into (3.17), we have

$$
u(x)=\int_{-1}^{1} G(x, y) f(y) d y \quad(-1<x<1)
$$

where

$$
\begin{aligned}
G(x, y)= & \frac{1}{2}\left[K_{0}(|x-y|)-\left(K_{\alpha_{0}}, K_{\alpha_{1}}\right)(x+1)\left(\begin{array}{ll}
K_{\alpha_{0}+\beta_{0}} & K_{\alpha_{1}+\beta_{0}} \\
K_{\alpha_{0}+\beta_{1}} & K_{\alpha_{1}+\beta_{1}}
\end{array}\right)^{-1}(2)\left(\begin{array}{c}
K_{\beta_{0}} \\
K_{\beta_{1}}
\end{array}\right)(1-y)\right. \\
& \left.+\left(K_{\beta_{0}}, K_{\beta_{1}}\right)(x-1)\left(\begin{array}{ll}
K_{\alpha_{0}+\beta_{0}} & K_{\alpha_{0}+\beta_{1}} \\
K_{\alpha_{1}+\beta_{0}} & K_{\alpha_{1}+\beta_{1}}
\end{array}\right)^{-1}(-2)\left(\begin{array}{c}
K_{\alpha_{0}} \\
K_{\alpha_{1}}
\end{array}\right)(-1-y)\right] .
\end{aligned}
$$


Since $K_{i}(-x)=(-1)^{i+1} K_{i}(x)$, we have

$$
\begin{gathered}
\left(K_{\beta_{0}}, K_{\beta_{1}}\right)(x-1)=\left(K_{\beta_{0}}, K_{\beta_{1}}\right)(1-x)\left(\begin{array}{cc}
(-1)^{\beta_{0}+1} & 0 \\
0 & (-1)^{\beta_{1}+1}
\end{array}\right) \\
\left(\begin{array}{ll}
K_{\alpha_{0}+\beta_{0}} & K_{\alpha_{0}+\beta_{1}} \\
K_{\alpha_{1}+\beta_{0}} & K_{\alpha_{1}+\beta_{1}}
\end{array}\right)(-2)=-\left(\begin{array}{cc}
(-1)^{\alpha_{0}+1} & 0 \\
0 & (-1)^{\alpha_{1}+1}
\end{array}\right)\left(\begin{array}{ll}
K_{\alpha_{0}+\beta_{0}} & K_{\alpha_{0}+\beta_{1}} \\
K_{\alpha_{1}+\beta_{0}} & K_{\alpha_{1}+\beta_{1}}
\end{array}\right)(2) \\
\times\left(\begin{array}{cc}
(-1)^{\beta_{0}+1} & 0 \\
0 & (-1)^{\beta_{1}+1}
\end{array}\right), \\
\left(\begin{array}{l}
K_{\alpha_{0}} \\
K_{\alpha_{1}}
\end{array}\right)(-1-y)=\left(\begin{array}{cc}
(-1)^{\alpha_{0}+1} & 0 \\
0 & (-1)^{\alpha_{1}+1}
\end{array}\right)\left(\begin{array}{l}
K_{\alpha_{0}} \\
K_{\alpha_{1}}
\end{array}\right)(1+y) .
\end{gathered}
$$

(3.22) is rewritten as

$$
\begin{aligned}
G(x, y)= & \frac{1}{2}\left[K_{0}(|x-y|)-\left(K_{\alpha_{0}}, K_{\alpha_{1}}\right)(1+x)\left(\begin{array}{cc}
K_{\alpha_{0}+\beta_{0}} & K_{\alpha_{1}+\beta_{0}} \\
K_{\alpha_{0}+\beta_{1}} & K_{\alpha_{1}+\beta_{1}}
\end{array}\right)^{-1}\left(\begin{array}{c}
K_{\beta_{0}} \\
K_{\beta_{1}}
\end{array}\right)(1-y)\right. \\
& \left.-\left(K_{\alpha_{0}}, K_{\alpha_{1}}\right)(1+y)\left(\begin{array}{cc}
K_{\alpha_{0}+\beta_{0}} & K_{\alpha_{1}+\beta_{0}} \\
K_{\alpha_{0}+\beta_{1}} & K_{\alpha_{1}+\beta_{1}}
\end{array}\right)^{-1}\left(\begin{array}{c}
K_{\beta_{0}} \\
K_{\beta_{1}}
\end{array}\right)(1-x)\right],
\end{aligned}
$$

where $K_{j}=K_{j}(2)$. The equivalence between the above expression and (3.3) is shown from the following well-known fact, that is, for any $N \times N$ regular matrix $\boldsymbol{A}$ and $N \times 1$ matrices $\boldsymbol{a}$ and $\boldsymbol{b}$, the equality

$$
{ }^{t} \boldsymbol{a} A^{-1} b=-\left|\begin{array}{l|l}
\boldsymbol{A} & \boldsymbol{b} \\
\hline{ }^{t} \boldsymbol{a} & 0
\end{array}\right| /|\boldsymbol{A}|
$$

holds. Inserting (3.1) into (3.3), we have (1.10) (1.15) in Theorem 1.1(2).

CASE II $(\alpha, \beta)=(0,2,2,3)(3.20)$ and (3.21) are rewritten as

$$
\begin{aligned}
& \left(\begin{array}{cc}
K_{2} & 0 \\
K_{3} & 0
\end{array}\right)(2)\left(\begin{array}{l}
u_{3} \\
u_{1}
\end{array}\right)(-1)=-\int_{-1}^{1}\left(\begin{array}{l}
K_{2} \\
K_{3}
\end{array}\right)(1-y) f(y) d y \\
& \left(\begin{array}{cc}
K_{2} & K_{3} \\
0 & 0
\end{array}\right)(-2)\left(\begin{array}{l}
u_{1} \\
u_{0}
\end{array}\right)(1)=\int_{-1}^{1}\left(\begin{array}{l}
K_{0} \\
K_{2}
\end{array}\right)(-1-y) f(y) d y .
\end{aligned}
$$

From the 1st row of (3.24), we have the solvability condition (3.4) and

$$
\begin{aligned}
& u_{3}(-1)=-\int_{-1}^{1} \frac{1}{K_{2}} K_{2}(1-y) f(y) d y=-\int_{-1}^{1} \frac{1}{K_{3}} K_{3}(1-y) f(y) d y, \\
& u_{1}(1)=\frac{K_{3}}{K_{2}} u_{0}(1)+\int_{-1}^{1} \frac{1}{K_{2}} K_{0}(1+y) f(y) d y .
\end{aligned}
$$


Substituting these equations into (3.17), we have (3.5) where

$$
G_{0}(x, y)=\frac{1}{2}\left[K_{0}(|x-y|)-\frac{1}{K_{2}}\left\{K_{0}(1+x) K_{2}(1-y)+K_{2}(1-x) K_{0}(1+y)\right\}\right],
$$

from which we obtain (3.6), or equivalently (3.7).

CASE II $(\alpha, \beta)=(1,3,1,3)(3.20)$ and (3.21) are rewritten as

$$
\begin{aligned}
& \left(\begin{array}{cc}
K_{2} & 0 \\
0 & 0
\end{array}\right)(2)\left(\begin{array}{l}
u_{2} \\
u_{0}
\end{array}\right)(-1)=-\int_{-1}^{1}\left(\begin{array}{l}
K_{1} \\
K_{3}
\end{array}\right)(1-y) f(y) d y \\
& \left(\begin{array}{cc}
K_{2} & 0 \\
0 & 0
\end{array}\right)(-2)\left(\begin{array}{l}
u_{2} \\
u_{0}
\end{array}\right)(1)=\int_{-1}^{1}\left(\begin{array}{l}
K_{1} \\
K_{3}
\end{array}\right)(-1-y) f(y) d y .
\end{aligned}
$$

From above, we have (3.4) and

$$
u_{2}(-1)=-\int_{-1}^{1} \frac{1}{K_{2}} K_{1}(1-y) f(y) d y, \quad u_{2}(1)=-\int_{-1}^{1} \frac{1}{K_{2}} K_{1}(1+y) f(y) d y .
$$

Substituting these equations into (3.17), we have (3.5) where

$$
G_{0}(x, y)=\frac{1}{2}\left[K_{0}(|x-y|)-\frac{1}{K_{2}}\left\{K_{1}(1+x) K_{1}(1-y)+K_{1}(1-x) K_{1}(1+y)\right\}\right],
$$

from which we obtain (3.6), or equivalently (3.8).

CASE II $(\alpha, \beta)=(1,3,2,3)(3.20)$ and (3.21) are rewritten as

$$
\begin{aligned}
& \left(\begin{array}{cc}
K_{3} & 0 \\
0 & 0
\end{array}\right)(2)\left(\begin{array}{l}
u_{2} \\
u_{0}
\end{array}\right)(-1)=-\int_{-1}^{1}\left(\begin{array}{l}
K_{2} \\
K_{3}
\end{array}\right)(1-y) f(y) d y \\
& \left(\begin{array}{cc}
K_{3} & 0 \\
0 & 0
\end{array}\right)(-2)\left(\begin{array}{l}
u_{1} \\
u_{0}
\end{array}\right)(1)=\int_{-1}^{1}\left(\begin{array}{l}
K_{1} \\
K_{3}
\end{array}\right)(-1-y) f(y) d y .
\end{aligned}
$$

From above, we have (3.4) and

$$
u_{2}(-1)=-\int_{-1}^{1} \frac{1}{K_{3}} K_{2}(1-y) f(y) d y, \quad u_{1}(1)=\int_{-1}^{1} \frac{1}{K_{3}} K_{1}(1+y) f(y) d y .
$$

Substituting these equations into (3.17), we have (3.5) where

$$
G_{0}(x, y)=\frac{1}{2}\left[K_{0}(|x-y|)-\frac{1}{K_{3}}\left\{K_{1}(1+x) K_{2}(1-y)+K_{2}(1-x) K_{1}(1+y)\right\}\right],
$$

from which we obtain (3.6), or equivalently (3.9). 
CASE III From (3.20) and (3.21), we have

$$
\int_{-1}^{1} f(y)=0, \quad \int_{-1}^{1} y f(y)=0
$$

or equivalently (3.10). From (3.17), we have (3.11) and (3.12).

\section{Green Function}

In this section, we give a proof of Theorem 1.1 and investigate the properties of Green function. The important aim of this section is to construct unique Green functions in cases II and III.

The advantage of the method of symmetric orthogonalization is as follows. The orthogonality (Theorem $4.1(5))$ and the symmetry $(G(x, y)=G(y, x))$ assure the uniqueness of the solution. Moreover, thus obtained Green function is a reproducing kernel as shown in section 5 .

Starting from the proto Green function $G_{0}(x, y)$, we can construct Green function $G(x, y)$ which has both symmetric and orthogonal properties, as is shown later in Lemma 4.2 and Lemma 4.3. We call this procedure generating $G(x, y)$ from $G_{0}(x, y)$ "the method of symmetric orthogonalization".

We start with the following lemma, which plays an important role in performing the symmetric orthogonalization method.

\section{LEMMA 4.1.}

$$
\begin{gathered}
\int_{-1}^{1} K_{0}(|x-y|) d y=K_{-1}(1+x)+K_{-1}(1-x)=\frac{1}{12}\left[x^{4}+6 x^{2}+1\right] \\
\int_{-1}^{1} K_{0}(|x-y|)(1+y) d y=K_{-2}(1+x)-K_{-2}(1-x)+2 K_{-1}(1-x) \\
=\frac{1}{60}\left[x^{5}+5 x^{4}-10 x^{3}+30 x^{2}-15 x+5\right] \\
\int_{-1}^{1} K_{0}(|x-y|) y d y=K_{-2}(1+x)-K_{-2}(1-x)-K_{-1}(1+x)+K_{-1}(1-x) \\
=\frac{1}{60}\left[x^{5}-10 x^{3}-15 x\right] \\
\int_{-1}^{1} K_{j}(1+y) d y=\int_{-1}^{1} K_{j}(1-y) d y=K_{j-1} \quad(j \neq 4)
\end{gathered}
$$




$$
\begin{aligned}
& \int_{-1}^{1} K_{j}(1+y)(1+y) d y=2 K_{j-1}-K_{j-2} \quad(j \neq 5) \\
& \int_{-1}^{1} K_{j}(1-y)(1+y) d y=K_{j-2} \quad(j \neq 4,5)
\end{aligned}
$$

Since the above lemma is shown through direct calculations, we omit its proof.

LEMma 4.2 (CASE II). For any bounded continuous function $f(x)$ $(-1<x<1)$ satisfying

$$
\int_{-1}^{1} f(y) \varphi(y) d y=0
$$

the boundary value problem

$$
\begin{cases}u^{(4)}=f(x) & (-1<x<1) \\ u^{\left(\alpha_{i}\right)}(-1)=u^{\left(\beta_{i}\right)}(1)=0 & (i=0,1) \\ \int_{-1}^{1} u(x) \varphi(x) d x=0 & \end{cases}
$$

has a unique classical solution $u(x)$ expressed as

$$
u(x)=\int_{-1}^{1} G(x, y) f(y) d y \quad(-1<x<1)
$$

where $G(x, y)=G(\alpha, \beta ; x, y)$ are Green functions which are constructed by the following formula:

$$
G(x, y)=G_{0}(x, y)-\psi(x) \varphi(y)-\psi(y) \varphi(x)+g \varphi(x) \varphi(y) \quad(-1<x, y<1)
$$

where

$$
\begin{aligned}
& \psi(x)=\int_{-1}^{1} G_{0}(x, y) \varphi(y) d y \quad(-1<x<1) \\
& g=\int_{-1}^{1} \int_{-1}^{1} \varphi(x) G_{0}(x, y) \varphi(y) d y d x=\int_{-1}^{1} \varphi(x) \psi(x) d x \\
& =\int_{-1}^{1} \psi(y) \varphi(y) d y .
\end{aligned}
$$


$\varphi(x)=\varphi(\alpha, \beta ; x)$ is the normalized eigenfunction of $\operatorname{EVP}(\alpha, \beta)$ in (1.5) corresponding to the eigen value $\lambda=0$. The concrete formulae of $G(x, y)$ are given by $(1.16) \sim(1.18)$.

Proof of Lemma 4.2. It is easy to show $\int_{-1}^{1} \varphi(x) G(x, y) d x=0$ for any fixed $y(-1 \leq y \leq 1)$. Hence, $\int_{-1}^{1} \varphi(x) u(x) d x=0$ holds. From Theorem 3.1, (4.7) is a unique solution to $\operatorname{BVP}(\alpha, \beta)$.

Let us calculate the concrete forms of Green function.

Proof OF THEOREM 1.1(2) (1.16) (1.18). It is enough to find concrete forms of $\psi(x)$ and $g$, which are obtained by substituting (3.6) into (4.9) and (4.10). $(\alpha, \beta)=(0,2,2,3) \quad$ Using (4.2), (4.5) and (4.6), we have

$$
\begin{aligned}
& \psi(x)=\int_{-1}^{1} G_{0}(x, y) \varphi(y) d y=\frac{1}{2} \sqrt{\frac{3}{8}}\left[K_{-2}(1+x)-K_{-2}(1-x)+2 K_{-1}(1-x)\right. \\
& \left.+K_{2}^{-1}\left\{\left|\begin{array}{c|c}
K_{2} & K_{0} \\
\hline K_{0}(1+x) & 0
\end{array}\right|+\left|\begin{array}{c|c}
K_{2} & K_{2}(1-x) \\
\hline 2 K_{-1}-K_{-2} & 0
\end{array}\right|\right\}\right] \\
& =\frac{1}{360} \sqrt{\frac{3}{8}}\left[3 x^{5}+15 x^{4}-50 x^{3}+30 x^{2}-9 x-101\right], \\
& g=\int_{-1}^{1} \psi(x) \varphi(x) d x=\frac{3}{8}\left[2 K_{-3}-K_{-4}+K_{2}^{-1}\left|\frac{K_{2}}{2 K_{-1}-K_{-2}}\right| \frac{K_{0}}{0} \mid\right]=-\frac{22}{105} . \\
& (\alpha, \beta)=(1,3,1,3) \quad \text { Using (4.1) and (4.4), we have } \\
& \psi(x)=\int_{-1}^{1} G_{0}(x, y) \varphi(y) d y=\frac{1}{2 \sqrt{2}}\left[K_{-1}(1+x)+K_{-1}(1-x)\right. \\
& \left.+K_{2}^{-1}\left\{\left|\begin{array}{c|c}
K_{2} & K_{0} \\
\hline K_{1}(1+x) & 0
\end{array}\right|+\left|\begin{array}{c|c}
K_{2} & K_{1}(1-x) \\
\hline K_{0} & 0
\end{array}\right|\right\}\right]=\frac{1}{24 \sqrt{2}}\left[x^{4}-2 x^{2}-7\right], \\
& g=\int_{-1}^{1} \psi(x) \varphi(x) d x=\frac{1}{2}\left[K_{-2}+K_{2}^{-1}\left|\begin{array}{c|c}
K_{2} & K_{0} \\
\hline K_{0} & 0
\end{array}\right|\right]=-\frac{14}{45} . \\
& (\alpha, \beta)=(1,3,2,3) \quad \text { Using (4.1) and (4.4), we have }
\end{aligned}
$$




$$
\begin{aligned}
& \psi(x)=\int_{-1}^{1} G_{0}(x, y) \varphi(y) d y=\frac{1}{2 \sqrt{2}}\left[K_{-1}(1+x)+K_{-1}(1-x)\right. \\
& \left.+K_{3}^{-1}\left\{\left|\begin{array}{c|c}
K_{3} & K_{1} \\
\hline K_{1}(1+x) & 0
\end{array}\right|+\left|\begin{array}{c|c}
K_{3} & K_{2}(1-x) \\
\hline K_{0} & 0
\end{array}\right|\right\}\right] \\
& =\frac{1}{24 \sqrt{2}}\left[x^{4}-6 x^{2}-8 x-27\right] \text {, } \\
& g=\int_{-1}^{1} \psi(x) \varphi(x) d x=\frac{1}{2}\left[K_{-2}+K_{3}^{-1}\left|\begin{array}{|c|c}
K_{3} & K_{1} \\
\hline K_{0} & 0
\end{array}\right|\right]=-\frac{6}{5} .
\end{aligned}
$$

In the above three cases, inserting the above $\psi(x)$ and $g$ into (4.8), we obtain $(1.16) \sim(1.18)$.

LEMma 4.3 (CASE III). For any bounded continuous function $f(x)$ $(-1<x<1)$ satisfying

$$
\int_{-1}^{1} f(y) \varphi_{i}(y) d y=0 \quad(i=0,1),
$$

the boundary value problem

$$
\begin{cases}u^{(4)}=f(x) & (-1<x<1) \\ u^{\left(\alpha_{i}\right)}(-1)=u^{\left(\beta_{i}\right)}(1)=0 & (i=0,1) \\ \int_{-1}^{1} u(x) \varphi_{i}(x) d x=0 & (i=0,1)\end{cases}
$$

has a unique classical solution $u(x)$ expressed as

$$
u(x)=\int_{-1}^{1} G(x, y) f(y) d y \quad(-1<x<1)
$$

where $G(x, y)=G(\alpha, \beta ; x, y)$ is Green function which can be constructed by the following formula:

$$
\begin{aligned}
G(x, y)= & G_{0}(x, y)-\sum_{i=0}^{1}\left[\psi_{i}(x) \varphi_{i}(y)+\psi_{i}(y) \varphi_{i}(x)\right] \\
& +\sum_{i, j=0}^{1} g_{i j} \varphi_{i}(x) \varphi_{j}(y) \quad(-1<x, y<1)
\end{aligned}
$$


where

$$
\begin{aligned}
& \psi_{i}(x)=\int_{-1}^{1} G_{0}(x, y) \varphi_{i}(y) d y \quad(i=0,1,-1<x<1) \\
& g_{i j}=\int_{-1}^{1} \int_{-1}^{1} \varphi_{i}(x) G_{0}(x, y) \varphi_{j}(y) d y d x=\int_{-1}^{1} \varphi_{i}(x) \psi_{j}(x) d x \\
& =\int_{-1}^{1} \psi_{i}(y) \varphi_{j}(y) d y \quad(i, j=0,1) .
\end{aligned}
$$

$\varphi_{i}(x)$ is the normalized eigenfunction of $\operatorname{EVP}(\alpha, \beta)$ in (1.6) corresponding to the eigen value $\lambda=0$. The concrete formula of $G(x, y)$ is given by (1.19).

Proof of Lemma 4.3. It is easy to show $\int_{-1}^{1} \varphi_{i}(x) G(x, y) d x=0$ for any fixed $y(-1 \leq y \leq 1)$. Hence, $\int_{-1}^{1} \varphi_{i}(x) u(x) d x=0$ holds. From Theorem 3.1, (4.11) is a unique solution to $\operatorname{BVP}(2,3,2,3)$.

Let us calculate concrete forms of Green function.

Proof of Theorem 1.1(2) (1.19). Substituting (3.12) into (4.13) and (4.14), we have

$$
\begin{aligned}
& \psi_{0}(x)=\frac{1}{24 \sqrt{2}}\left[x^{4}+6 x^{2}+1\right], \quad \psi_{1}(x)=\frac{1}{120} \sqrt{\frac{3}{2}}\left[x^{5}-10 x^{3}-15 x\right], \\
& g_{00}=\frac{2}{15}, \quad g_{01}=g_{10}=0, \quad g_{11}=-\frac{6}{35} .
\end{aligned}
$$

Inserting the above $\psi_{i}(x)$ and $g_{i j}$ into (4.12), we obtain (1.19).

THEOREM 4.1. Green functions $G(x, y)=G(\alpha, \beta ; x, y)$ satisfy the following properties:

(1) $\partial_{x}^{4} G(x, y)$

$$
\begin{aligned}
& =\left\{\begin{array}{lr}
0 & (\alpha, \beta)=(0,1,0,1),(0,1,0,2),(0,1,1,3), \\
& (0,1,2,3),(0,2,0,2),(0,2,1,3) \\
-\varphi(\alpha, \beta ; x) \varphi(\alpha, \beta ; y) & (\alpha, \beta)=(0,2,2,3),(1,3,1,3),(1,3,2,3) \\
-\varphi_{0}(x) \varphi_{0}(y)-\varphi_{1}(x) \varphi_{1}(y) & (\alpha, \beta)=(2,3,2,3)
\end{array}\right. \\
& \begin{aligned}
(-1<x, y<1, x \neq y) & (i=0,1,-1<y<1)
\end{aligned}
\end{aligned}
$$


(3) $\left.\partial_{x}^{i} G(x, y)\right|_{y=x-0}-\left.\partial_{x}^{i} G(x, y)\right|_{y=x+0}= \begin{cases}0 & (0 \leq i \leq 2) \\ 1 & (i=3) \quad(-1<x<1)\end{cases}$

(4) $\left.\partial_{x}^{i} G(x, y)\right|_{x=y+0}-\left.\partial_{x}^{i} G(x, y)\right|_{x=y-0}= \begin{cases}0 & (0 \leq i \leq 2) \\ 1 & (i=3) \quad(-1<y<1)\end{cases}$

(5) $\int_{-1}^{1} \varphi(\alpha, \beta ; x) G(x, y) d x=0 \quad(\alpha, \beta)=(0,2,2,3),(1,3,1,3),(1,3,2,3)$

$$
\int_{-1}^{1} \varphi_{i}(x) G(x, y) d x=0 \quad(i=0,1), \quad(\alpha, \beta)=(2,3,2,3) \quad(-1<y<1) .
$$

Proof of Theorem 4.1. We give concrete forms of $i$-th derivative $(i=0,1$, 2,3,4) of Green functions in Appendix, from which (1), (2), (3) are derived. (4) follows from (3). (5) is given by (4.8) and (4.12). Thus we have Theorem 4.1 .

Proof of Theorem 1.1(1). The uniqueness of the solution was shown by Theorem 3.1, Lemma 4.2 and Lemma 4.3. Differentiating $u(x)(-1<x<1)$ in (1.9) $i(0 \leq i \leq 4)$ times and using Theorem 4.1 (1), (2), (3) and (5), we can show that the existence of the solution.

\section{Reproducing Kernel}

In this section, it is shown that Green function $G(x, y)$ is a reproducing kernel for a set of Hilbert space $H$ and its inner product $(\cdot, \cdot)_{H}$, which is introduced in section 2 .

THEOREM 5.1. (1) For any $u(x) \in H$, we have the reproducing relation

$$
u(y)=(u(x), G(x, y))_{H}=\int_{-1}^{1} u^{\prime \prime}(x) \partial_{x}^{2} G(x, y) d x \quad(-1 \leq y \leq 1) .
$$

This means that Green function $G(x, y)$ is a reproducing kernel for $\left\{H,(\cdot, \cdot)_{H}\right\}$.

(2) $G(y, y)=\int_{-1}^{1}\left|\partial_{x}^{2} G(x, y)\right|^{2} d x \quad(-1 \leq y \leq 1)$.

Proof of TheOrem 5.1. For functions $u=u(x) \in H$ and $v=v(x)=G(x, y)$ with $y$ arbitrarily fixed in $-1 \leq y \leq 1$, integrating the identity

$$
u^{\prime \prime} v^{\prime \prime}=\left[u^{\prime} v^{\prime \prime}-u v^{\prime \prime \prime}\right]^{\prime}+u v^{(4)}
$$


with respect to $x$ on intervals $-1<x<y$ and $y<x<1$, we have

$$
\begin{aligned}
& \int_{-1}^{1} u^{\prime \prime}(x) v^{\prime \prime}(x) d x \\
& =\left[u^{\prime}(x) v^{\prime \prime}(x)-u(x) v^{\prime \prime \prime}(x)\right]\left\{\left.\right|_{x=-1} ^{x=y-0}+\left.\right|_{x=y+0} ^{x=1}\right\}+\int_{-1}^{1} u(x) v^{(4)}(x) d x \\
& =u^{\prime}(1) v^{\prime \prime}(1)-u(1) v^{\prime \prime \prime}(1)-u^{\prime}(-1) v^{\prime \prime}(-1)+u(-1) v^{\prime \prime \prime}(-1) \\
& \quad+u^{\prime}(y)\left[v^{\prime \prime}(y-0)-v^{\prime \prime}(y+0)\right]-u(y)\left[v^{\prime \prime \prime}(y-0)-v^{\prime \prime \prime}(y+0)\right] \\
& \quad+\int_{-1}^{1} u(x) v^{(4)}(x) d x=u(y)
\end{aligned}
$$

In the last equality, we have employed Theorem 4.1. This proves (1). (2) follows from (1) by putting $u(x)=G(x, y)$ in (5.1). We have proved Theorem 5.1 .

\section{Sobolev Inequality}

In this section, we give a proof of Theorem 2.1(1).

Proof of Theorem 2.1(1). Applying Schwarz inequality to (5.1) and using (5.2), we have

$$
|u(y)|^{2} \leq \int_{-1}^{1}\left|\partial_{x}^{2} G(x, y)\right|^{2} d x \int_{-1}^{1}\left|u^{\prime \prime}(x)\right|^{2} d x=G(y, y) \int_{-1}^{1}\left|u^{\prime \prime}(x)\right|^{2} d x .
$$

Noting that $C(\alpha, \beta)=\max _{|y| \leq 1} G(y, y)=G\left(y_{0}, y_{0}\right)$, we have Sobolev inequality

$$
\left(\sup _{|y| \leq 1}|u(y)|\right)^{2} \leq C(\alpha, \beta) \int_{-1}^{1}\left|u^{\prime \prime}(x)\right|^{2} d x .
$$

This inequality shows that $(\cdot, \cdot)_{H}$ is positive definite. It should be noted that it requires Schwarz inequality but does not require "positive definiteness" of the inner product to prove (6.1).

In the second place, we apply this inequality to $u(x)=G\left(x, y_{0}\right) \in H$ and have

$$
\left(\sup _{|y| \leq 1}\left|G\left(y, y_{0}\right)\right|\right)^{2} \leq C(\alpha, \beta) \int_{-1}^{1}\left|\partial_{x}^{2} G\left(x, y_{0}\right)\right|^{2} d x=(C(\alpha, \beta))^{2} .
$$


Combining this and trivial inequality

$$
(C(\alpha, \beta))^{2}=\left(G\left(y_{0}, y_{0}\right)\right)^{2} \leq\left(\sup _{|y| \leq 1}\left|G\left(y, y_{0}\right)\right|\right)^{2},
$$

we have

$$
(C(\alpha, \beta))^{2} \leq\left(\sup _{|y| \leq 1}\left|G\left(y, y_{0}\right)\right|\right)^{2} \leq C(\alpha, \beta) \int_{-1}^{1}\left|\partial_{x}^{2} G\left(x, y_{0}\right)\right|^{2} d x=(C(\alpha, \beta))^{2} .
$$

Hence we obtain

$$
\left(\sup _{|y| \leq 1}\left|G\left(y, y_{0}\right)\right|\right)^{2}=C(\alpha, \beta) \int_{-1}^{1}\left|\partial_{x}^{2} G\left(x, y_{0}\right)\right|^{2} d x
$$

which completes the proof of Theorem 2.1(1).

\section{The Best Constant of Sobolev Inequality}

In this section, we calculate the best constant $C(\alpha, \beta)$ in Theorem 2.1(2), which is given by

$$
C(\alpha, \beta)=\max _{|y| \leq 1}|G(\alpha, \beta ; y, y)|=\max _{|y| \leq 1} G(\alpha, \beta ; y, y) .
$$

It should be noted that from (5.2) diagonal values $G(\alpha, \beta ; y, y)(-1 \leq y \leq 1)$ are non-negative.

Proof of THEOREM 2.1(2).

(1) $(\alpha, \beta)=(0,1,0,1)$ : Since $G(y, y)=\frac{1}{24}\left(1-y^{2}\right)^{3}$, we have $C(0,1,0,1)=$ $G(0,0)=\frac{1}{24}$.

(2) $(\alpha, \beta)=(0,1,0,2):$ Since

$$
\begin{aligned}
& G(y, y)=\frac{1}{96}\left[-y^{6}+6 y^{5}+9 y^{4}-12 y^{3}-15 y^{2}+6 y+7\right] \\
&= \frac{1}{96}(7-y)(1-y)^{2}(1+y)^{3} \\
& \frac{d}{d y} G(y, y)=\frac{1}{16}(1-y)(1+y)^{2}\left(y^{2}-6 y+1\right) \begin{cases}>0 & \left(-1<y<y_{0}\right) \\
=0 & \left(y=y_{0}\right) \\
<0 & \left(y_{0}<y<1\right)\end{cases}
\end{aligned}
$$


where $y_{0}=3-2 \sqrt{2}$, we have

$$
C(0,1,0,2)=G\left(y_{0}, y_{0}\right)=\frac{8}{3}(17-12 \sqrt{2}) .
$$
have

(3) $(\alpha, \beta)=(0,1,1,3):$ Since $G(y, y)=\frac{1}{24}\left[-3 y^{4}-4 y^{3}+6 y^{2}+12 y+5\right]$, we

$$
G(1,1)-G(y, y)=\frac{1}{24}(1-y)^{2}\left[3\left(y+\frac{5}{3}\right)^{2}+\frac{8}{3}\right] \geq 0 \quad(-1 \leq y \leq 1)
$$

and therefore $C(0,1,1,3)=G(1,1)=\frac{2}{3}$.

(4) $(\alpha, \beta)=(0,1,2,3)$ : Since $G(y, y)=\frac{1}{3}(1+y)^{3}$, we have $C(0,1,2,3)=$ $G(1,1)=\frac{8}{3}$.

(5) $(\alpha, \beta)=(0,2,0,2)$ : Since $G(y, y)=\frac{1}{6}\left(1-y^{2}\right)^{2}$, we have $C(0,2,0,2)=$ $G(0,0)=\frac{1}{6}$.

(6) $(\alpha, \beta)=(0,2,1,3)$ : Since $G(y, y)=\frac{2}{3}\left[-y^{3}+3 y+2\right]$, we have

$$
G(1,1)-G(y, y)=\frac{2}{3}(1-y)^{2}(2+y) \geq 0 \quad(-1 \leq y \leq 1)
$$

and therefore $C(0,2,1,3)=G(1,1)=\frac{8}{3}$.

(7) $(\alpha, \beta)=(0,2,2,3):$ Since $G(y, y)=\frac{1}{3360}\left[-21 y^{6}-126 y^{5}+525 y^{4}+700 y^{3}\right.$ $\left.-411 y^{2}-318 y+163\right]$ we have

$$
\begin{aligned}
G(1,1)-G(y, y)= & \frac{1}{3360}(1-y)\left[-21 y^{5}-147 y^{4}+378 y^{3}+1078 y^{2}+667 y+349\right] \\
= & \frac{1}{3360}(1-y)\left[21\left(1-y^{5}\right)+147\left(1-y^{4}\right)+378 y^{2}(1+y)\right. \\
& \left.+700\left(y+\frac{667}{1400}\right)^{2}+\frac{61911}{2800}\right] \geq 0 \quad(-1 \leq y \leq 1)
\end{aligned}
$$

and therefore $C(0,2,2,3)=G(1,1)=\frac{16}{105}$.

(8) $(\alpha, \beta)=(1,3,1,3):$ Since $G(y, y)=\frac{1}{90}\left[16-15\left(1-y^{2}\right)^{2}\right]$, we have

$$
C(1,3,1,3)=G(-1,-1)=G(1,1)=\frac{8}{45} .
$$


(9) $(\alpha, \beta)=(1,3,2,3)$ : Since $G(y, y)=\frac{1}{120}\left[-5 y^{4}+60 y^{3}+90 y^{2}-20 y+3\right]$, we have

$$
\begin{aligned}
G(1,1)-G(y, y) & =\frac{1}{24}(1-y)\left[y^{2}(1-y)+10\left(y+\frac{29}{20}\right)^{2}+\frac{159}{40}\right] \\
& \geq 0 \quad(-1 \leq y \leq 1)
\end{aligned}
$$

and therefore $C(1,3,2,3)=G(1,1)=\frac{16}{15}$.

we have

$(10)(\alpha, \beta)=(2,3,2,3):$ Since $G(y, y)=\frac{1}{840}\left[-21 y^{6}+175 y^{4}-111 y^{2}+21\right]$,

$$
G(1,1)-G(y, y)=\frac{1}{840}\left(1-y^{2}\right)\left[43+133 y^{2}+21 y^{2}\left(1-y^{2}\right)\right] \geq 0 \quad(-1 \leq y \leq 1)
$$

and therefore $C(2,3,2,3)=G(-1,-1)=G(1,1)=\frac{8}{105}$. This completes the proof of Theorem 2.1(2).

\section{Acknowledgment}

One of the authors (K. T.) is supported by J. S. P. S. Grant-in-Aid for Scientific Research (C) No. 21540148.

\section{Appendix}

We have list $G(x, y)$ and its $i$-th derivatives $(1 \leq i \leq 4)$, which are used in the proof of Theorem 4.1. We remark $-1<x, y<1$ and $x \neq y$.

(1) $G(x, y)=G(0,1,0,1 ; x, y)$

$$
\begin{aligned}
& G(x, y)= \frac{1}{12}|x-y|^{3}+\frac{1}{24}\left[-x^{3} y^{3}+3\left(x^{3} y+x y^{3}\right)-3 x^{2} y^{2}-3\left(x^{2}+y^{2}\right)\right. \\
&+3 x y+1] \\
& \partial_{x} G(x, y)= \frac{1}{4} \operatorname{sgn}(x-y)|x-y|^{2}+\frac{1}{8}\left[-x^{2} y^{3}+3 x^{2} y+y^{3}-2 x y^{2}-2 x+y\right] \\
& \partial_{x}^{2} G(x, y)= \frac{1}{2}|x-y|+\frac{1}{4}\left[-x y^{3}+3 x y-y^{2}-1\right] \\
& \partial_{x}^{3} G(x, y)= \frac{1}{2} \operatorname{sgn}(x-y)+\frac{1}{4}\left[-y^{3}+3 y\right] \\
& \partial_{x}^{4} G(x, y)=0 .
\end{aligned}
$$


(2) $G(x, y)=G(0,1,0,2 ; x, y)$

$$
\begin{aligned}
G(x, y)= & \frac{1}{12}|x-y|^{3}+\frac{1}{96}\left[-x^{3} y^{3}+3\left(x^{3} y^{2}+x^{2} y^{3}\right)+9\left(x^{3} y+x y^{3}\right)-9 x^{2} y^{2}\right. \\
- & \left.3\left(x^{3}+y^{3}\right)-3\left(x^{2} y+x y^{2}\right)-15\left(x^{2}+y^{2}\right)+15 x y+3(x+y)+7\right] \\
\partial_{x} G(x, y)= & \frac{1}{4} \operatorname{sgn}(x-y)|x-y|^{2}+\frac{1}{32}\left[-x^{2} y^{3}+3 x^{2} y^{2}+2 x y^{3}+9 x^{2} y\right. \\
& \left.+3 y^{3}-6 x y^{2}-3 x^{2}-2 x y-y^{2}-10 x+5 y+1\right] \\
\partial_{x}^{2} G(x, y)= & \frac{1}{2}|x-y|+\frac{1}{16}\left[-x y^{3}+3 x y^{2}+y^{3}+9 x y-3 y^{2}-3 x-y-5\right] \\
\partial_{x}^{3} G(x, y)= & \frac{1}{2} \operatorname{sgn}(x-y)+\frac{1}{16}\left[-y^{3}+3 y^{2}+9 y-3\right] \\
\partial_{x}^{4} G(x, y)= & 0 .
\end{aligned}
$$

(3) $G(x, y)=G(0,1,1,3 ; x, y)$

$$
\begin{aligned}
G(x, y)= & \frac{1}{12}|x-y|^{3}+\frac{1}{24}\left[-3 x^{2} y^{2}-2\left(x^{3}+y^{3}\right)-3\left(x^{2}+y^{2}\right)+12 x y\right. \\
& +6(x+y)+5]
\end{aligned}
$$$$
\partial_{x} G(x, y)=\frac{1}{4} \operatorname{sgn}(x-y)|x-y|^{2}+\frac{1}{4}\left[-x y^{2}-x^{2}-x+2 y+1\right]
$$$$
\partial_{x}^{2} G(x, y)=\frac{1}{2}|x-y|-\frac{1}{4}\left[y^{2}+2 x+1\right]
$$$$
\partial_{x}^{3} G(x, y)=\frac{1}{2}[\operatorname{sgn}(x-y)-1]
$$$$
\partial_{x}^{4} G(x, y)=0 .
$$

(4) $G(x, y)=G(0,1,2,3 ; x, y)$

$$
\begin{aligned}
& G(x, y)=\frac{1}{12}\left[|x-y|^{3}-\left(x^{3}+y^{3}\right)+3\left(x^{2} y+x y^{2}\right)+12 x y+6(x+y)+4\right] \\
& \partial_{x} G(x, y)=\frac{1}{4}\left[\operatorname{sgn}(x-y)|x-y|^{2}-x^{2}+2 x y+y^{2}+4 y+2\right] \\
& \partial_{x}^{2} G(x, y)=\frac{1}{2}[|x-y|-x+y] \\
& \partial_{x}^{3} G(x, y)=\frac{1}{2}[\operatorname{sgn}(x-y)-1] \\
& \partial_{x}^{4} G(x, y)=0 .
\end{aligned}
$$


(5) $G(x, y)=G(0,2,0,2 ; x, y)$

$$
\begin{aligned}
& G(x, y)=\frac{1}{12}\left[|x-y|^{3}+x^{3} y+x y^{3}-3\left(x^{2}+y^{2}\right)+2 x y+2\right] \\
& \partial_{x} G(x, y)=\frac{1}{12}\left[3 \operatorname{sgn}(x-y)|x-y|^{2}+3 x^{2} y+y^{3}-6 x+2 y\right] \\
& \partial_{x}^{2} G(x, y)=\frac{1}{2}[|x-y|+x y-1] \\
& \partial_{x}^{3} G(x, y)=\frac{1}{2}[\operatorname{sgn}(x-y)+y] \\
& \partial_{x}^{4} G(x, y)=0 .
\end{aligned}
$$

(6) $G(x, y)=G(0,2,1,3 ; x, y)$

$$
\begin{aligned}
G(x, y)= & \frac{1}{12}\left[|x-y|^{3}-\left(x^{3}+y^{3}\right)-3\left(x^{2} y+x y^{2}\right)-6\left(x^{2}+y^{2}\right)+12 x y\right. \\
& +12(x+y)+16]
\end{aligned}
$$$$
\partial_{x} G(x, y)=\frac{1}{4}\left[\operatorname{sgn}(x-y)|x-y|^{2}-x^{2}-2 x y-y^{2}-4 x+4 y+4\right]
$$$$
\partial_{x}^{2} G(x, y)=\frac{1}{2}[|x-y|-x-y-2]
$$$$
\partial_{x}^{3} G(x, y)=\frac{1}{2}[\operatorname{sgn}(x-y)-1]
$$$$
\partial_{x}^{4} G(x, y)=0 \text {. }
$$

(7) $G(x, y)=G(0,2,2,3 ; x, y)$

$$
\begin{aligned}
G(x, y)= & \frac{1}{12}|x-y|^{3}+\frac{1}{6720}\left[-21\left(x^{5} y+x y^{5}\right)-21\left(x^{5}+y^{5}\right)-105\left(x^{4} y+x y^{4}\right)\right. \\
& -105\left(x^{4}+y^{4}\right)+630\left(x^{3} y+x y^{3}\right)+70\left(x^{3}+y^{3}\right)+630\left(x^{2} y+x y^{2}\right) \\
& \left.-1050\left(x^{2}+y^{2}\right)+1278 x y-318(x+y)+326\right]
\end{aligned}
$$

$$
\begin{aligned}
\partial_{x} G(x, y)= & \frac{1}{4} \operatorname{sgn}(x-y)|x-y|^{2}+\frac{1}{2240}\left[-35 x^{4} y-7 y^{5}-35 x^{4}-140 x^{3} y\right. \\
& -35 y^{4}-140 x^{3}+630 x^{2} y+210 y^{3}+70 x^{2}+420 x y+210 y^{2} \\
& -700 x+426 y-106]
\end{aligned}
$$




$$
\begin{aligned}
& \partial_{x}^{2} G(x, y)=\frac{1}{2}|x-y|+\frac{1}{16}\left[-x^{3} y-x^{3}-3 x^{2} y-3 x^{2}+9 x y+x+3 y-5\right] \\
& \partial_{x}^{3} G(x, y)=\frac{1}{2} \operatorname{sgn}(x-y)+\frac{1}{16}\left[-3 x^{2} y-3 x^{2}-6 x y-6 x+9 y+1\right] \\
& \partial_{x}^{4} G(x, y)=-\frac{3}{8}(x+1)(y+1)=-\varphi(x) \varphi(y) . \\
& \text { (8) } G(x, y)=G(1,3,1,3 ; x, y)
\end{aligned}
$$$$
G(x, y)=\frac{1}{12}|x-y|^{3}-\frac{1}{48}\left[x^{4}+y^{4}+6 x^{2} y^{2}+4\left(x^{2}+y^{2}\right)-24 x y\right]+\frac{1}{90}
$$$$
\partial_{x} G(x, y)=\frac{1}{4} \operatorname{sgn}(x-y)|x-y|^{2}-\frac{1}{12}\left[x^{3}+3 x y^{2}+2 x-6 y\right]
$$$$
\partial_{x}^{2} G(x, y)=\frac{1}{2}|x-y|-\frac{1}{12}\left[3 x^{2}+3 y^{2}+2\right]
$$$$
\partial_{x}^{3} G(x, y)=\frac{1}{2}[\operatorname{sgn}(x-y)-x]
$$$$
\partial_{x}^{4} G(x, y)=-\frac{1}{2}=-\varphi(x) \varphi(y) .
$$

(9) $G(x, y)=G(1,3,2,3 ; x, y)$

$$
\begin{aligned}
& G(x, y)= \frac{1}{12}|x-y|^{3}+\frac{1}{48}\left[-\left(x^{4}+y^{4}\right)+12\left(x^{2} y+x y^{2}\right)-6\left(x^{2}+y^{2}\right)\right. \\
&+48 x y-4(x+y)]+\frac{1}{40} \\
& \partial_{x} G(x, y)= \frac{1}{4} \operatorname{sgn}(x-y)|x-y|^{2}+\frac{1}{12}\left[-x^{3}+6 x y+3 y^{2}-3 x+12 y-1\right] \\
& \partial_{x}^{2} G(x, y)= \frac{1}{2}|x-y|+\frac{1}{4}\left[-x^{2}+2 y-1\right] \\
& \partial_{x}^{3} G(x, y)= \frac{1}{2}[\operatorname{sgn}(x-y)-x] \\
& \partial_{x}^{4} G(x, y)=-\frac{1}{2}=-\varphi(x) \varphi(y) . \\
&(10) G(x, y)=G(2,3,2,3 ; x, y)
\end{aligned}
$$

$$
\begin{aligned}
G(x, y)= & \frac{1}{12}|x-y|^{3}-\frac{1}{1680}\left[21\left(x^{5} y+x y^{5}\right)+35\left(x^{4}+y^{4}\right)-210\left(x^{3} y+x y^{3}\right)\right. \\
& \left.+210\left(x^{2}+y^{2}\right)-198 x y\right]+\frac{1}{40}
\end{aligned}
$$




$$
\begin{aligned}
\partial_{x} G(x, y)= & \frac{1}{4} \operatorname{sgn}(x-y)|x-y|^{2}-\frac{1}{1680}\left[105 x^{4} y+21 y^{5}+140 x^{3}-630 x^{2} y\right. \\
& \left.-210 y^{3}+420 x-198 y\right] \\
\partial_{x}^{2} G(x, y)= & \frac{1}{2}|x-y|-\frac{1}{4}\left[x^{3} y+x^{2}-3 x y+1\right] \\
\partial_{x}^{3} G(x, y)= & \frac{1}{2} \operatorname{sgn}(x-y)-\frac{1}{4}\left[3 x^{2} y+2 x-3 y\right] \\
\partial_{x}^{4} G(x, y)= & -\frac{1}{2}-\frac{3}{2} x y=-\varphi_{0}(x) \varphi_{0}(y)-\varphi_{1}(x) \varphi_{1}(y) .
\end{aligned}
$$

\section{References}

[1] H. Brezis, Functional Analysis, Masson Editeur 1988.

[2] Y. Kametaka, K. Takemura, Y. Suzuki and A. Nagai, Positivity and hierarchical structure of Green's functions of 2-point boundary value problems for bending of a beam, Japan $\mathbf{J}$. Ind. Appl. Math., 21 (2001), 543-566.

[ 3 ] Y. Kametaka, K. Watanabe, A. Nagai, H. Yamagishi and K. Takemura, The best constant of Sobolev inequality which correspond to a bending problem of a string with periodic boundary condition, Sci. Math. Jpn. e-2007 (2007), 283-300.

[4] Y. Kametaka, H. Yamagishi, K. Watanabe, A. Nagai and K. Takemura, Riemann-zeta function, Bernoulli polynomials and the best constant of Sobolev inequalities, Sci. Math. Jpn. e-2007 (2007), 63-89.

[ 5 ] Y. Kametaka, H. Yamagishi, K. Watanabe, A. Nagai and K. Takemura, Riemann zeta function and the best constants of three series of Sobolev inequalities, Transactions of the Japan Society for Industrial and Applied Mathematics 18 (2008), 29-40 [in Japanese].

[6] Y. Kametaka, H. Yamagishi, K. Watanabe, A. Nagai and K. Takemura, The best constant of Sobolev inequality corresponding to Dirichlet boundary value problem for $(-1)^{M}(d / d x)^{2 M}$, Sci. Math. Jpn. e-2008 (2008), 439-451.

[7] Y. Kametaka, H. Yamagishi, K. Watanabe, A. Nagai, K. Takemura and M. Arai, The best constant of some Sobolev inequality which corresponds to a Schrödinger operator with Dirac delta potential, Sci. Math. Jpn. e-2008 (2008), 541-555.

[ 8 ] Y. A. Melnikov, Influence functions and matrices, Mercel Dekker, New York, 1999.

[9] A. Nagai, K. Takemura, Y. Kametaka, K. Watanabe and H. Yamagishi, Green function for boundary value problem of $2 M$-th linear ordinary differential equations with free boundary condition, Far East J. Appl. Math., 26 (2007), 393-406.

[10] K. Takemura, A. Nagai, Y. Kametaka, K. Watanabe and H. Yamagishi, The best constant of Sobolev inequality corresponding to the free boundary value problem for $(-1)^{M}(d / d x)^{2 M}$, Transactions of the Japan Society for Industrial and Applied Mathematics 18 (2008), 29-40 [in Japanese].

[11] S. P. Timoshenko and J. N. Goodier: Theory of Elasticity (in Japanese), Corona, 1976.

[12] K. Watanabe, T. Yamada and W. Takahashi, Reproducing Kernels of $H^{m}(a, b)(m=1,2,3)$ and Least Constants in Sobolev's Inequalities, Appl. Anal. 82 (2003), 809-820.

[13] H. Yamagishi, Y. Kametaka, A. Nagai, K. Watanabe and K. Takemura, Riemann zeta function and the best constants of five series of Sobolev inequalities, to appear in RIMS Kokyuroku Bessatsu. 
Kazuo Takemura

Liberal Arts and Basic Sciences, College of Industrial Technology

Nihon University, 2-11-1 Shinei, Narashino 275-8576, Japan

E-mail address: takemura.kazuo@nihon-u.ac.jp

Hiroyuki Yamagishi

Tokyo Metropolitan College of Industrial Technology

1-10-40 Higashi-ooi, Shinagawa Tokyo 140-0011, Japan

E-mail: yamagisi@s.metro-cit.ac.jp

Yoshinori Kametaka

He has retired at March 2004, and now he is an

emeritus professor of Osaka University

Faculty of Engineering Science, Osaka University

1-3 Matikaneyamatyo, Toyonaka 560-8531, Japan

E-mail address: kametaka@sigmath.es.osaka-u.ac.jp

Kohtaro Watanabe

Department of Computer Science, National Defense Academy

1-10-20 Yokosuka 239-8686, Japan

E-mail address: wata@nda.ac.jp

Atsushi Nagai

Liberal Arts and Basic Sciences, College of Industrial Technology

Nihon University, 2-11-1 Shinei, Narashino 275-8576, Japan

E-mail address: nagai.atsushi@nihon-u.ac.jp 